\title{
Eosinophilic Gastrointestinal Diseases in Childhood
}

\author{
Eleni Koutri Alexandra Papadopoulou \\ Division on Gastroenterology, Hepatology and Nutrition, First Department of Pediatrics, University of Athens, \\ Children's Hospital "Agia Sofia", Athens, Greece
}

\section{Key Messages}

- Eosinophilic gastrointestinal diseases (EGIDs) are rare chronic, inflammatory disorders of the gastrointestinal (GI) tract with unknown long-term sequelae. The clinical presentation depends on the involved GI site as well as the extent and the depth of eosinophilic inflammation through the bowel wall.

- In the absence of biological markers, the diagnosis is based on clinical symptoms and on histological findings of eosinophilic inflammation, after the exclusion of a secondary cause of inflammation or a systemic disorder, which may be a challenging issue given the absence of strict histological criteria for EGID (beyond the esophagus) diagnosis.

- Treatment strategies depend on the involved site of the Gl tract. Maintenance treatment is often necessary to avoid relapses.

- Consensus recommendations on the diagnosis of EGID (beyond the esophagus), as well as randomized studies assessing the efficacy and safety of various treatment modalities, are urgently needed.

\section{Keywords}

Eosinophilic gastrointestinal diseases - Eosinophilic esophagitis - Eosinophilic gastritis - Eosinophilic gastroenteritis · Eosinophilic colitis

\begin{abstract}
Eosinophilic gastrointestinal diseases (EGIDs) comprise a group of chronic, inflammatory diseases of the gastrointestinal (GI) tract, that are characterized, clinically, by symptoms related to the dysfunction of the involved segment(s) of the Gl tract, and histologically, by dense eosinophilic inflammation, in the absence of an identifiable secondary cause. The group of EGIDs comprises eosinophilic esophagitis (EoE), eosinophilic gastritis (EG), eosinophilic gastroenteritis (EGE), and eosinophilic colitis (EC). EoE is the most common and the best described EGID compared to EG, EGE, and EC. The clinical presentation of the EGIDs differs depending on the location and the extent of the eosinophilic infiltration in the $\mathrm{Gl}$ tract, as well as its depth through the bowel wall. In the absence of biological markers, the diagnosis is based on the combination of clinical symptoms with the histological features of EGIDs, after the exclusion of secondary causes of eosinophilic inflammation of the Gl tract. Treatment is individualized and includes elimination diets (mainly empiric or elemental) and/ordrugs, according to the involved GI segment: proton pump inhibitors or local steroids in $\mathrm{EoE}$; local or oral systemic steroids in EG/EGE limited to the duodenum; oral
\end{abstract}

\section{KARGER}

(c) 2019 S. Karger AG, Basel

E-Mail karger@karger.com

www.karger.com/anm
Alexandra Papadopoulou

Division of Gastroenterology Hepatology and Nutrition, First Department of Pediatrics University of Athens, Children's Hospital "Agia Sofia”

Thivon and Papadiamantopoulou, GR-11527 Athens (Greece)

E-Mail a.papadopoulou@ paidon-agiasofia.gr 
systemic steroids in EGE with lower small intestine and/or colon involvement. In patients with EoE, maintenance treatment with lower doses may be considered following histological remission with the means of drugs. In patients treated with elimination diets, disease food triggers identified during food reintroduction need to be further eliminated. Esophageal stenosis despite medical treatment requires endoscopic dilation, while the use of thiopurines or anti-TNF drugs may be considered in refractory or steroid-dependent EGID (other than EoE). The aim of this review is to provide the available evidence on each of the above disorders, to aid clinicians to interpret the clinical manifestations and the laboratory findings and choose the best available treatment option.

(c) 2019 S. Karger AG, Basel

\section{Introduction}

Eosinophilic gastrointestinal diseases (EGIDs) are rare chronic inflammatory disorders characterized clinically by a variety of symptoms (Table 1) depending on the segment of the gastrointestinal (GI) tract involved and histologically by eosinophilic inflammation of different parts of the GI tract (Table 2), in the absence of an identifiable secondary cause (Table 3) [1].

EGIDs are subclassified into eosinophilic esophagitis (EoE), eosinophilic gastritis (EG), eosinophilic gastroenteritis (EGE), and eosinophilic colitis (EC) depending on whether the eosinophilic infiltration is limited to the esophagus, stomach, small intestine, and colon, respectively. In some occasions, multiple parts of the GI tract can be involved, simultaneously or sequentially.

The definition, epidemiology, clinical manifestations, histological features, and treatment options of each of the EGIDs follow below.

\section{Eosinophilic Esophagitis}

EoE is a chronic immune/antigen-mediated inflammatory disease of the esophagus characterized clinically by symptoms related to esophageal dysfunction and histologically by dense esophageal inflammation [2]. The incidence of EoE is 5.1/100,000 persons/year, while the prevalence is about 29.5 cases/100,000 inhabitants [3].

The disease is more common in males and in patients with atopic diseases [4], mainly due to food allergens and aeroallergens [5], although it occurs also in patients with no history of atopy. The most common food triggers of EoE are milk, followed by wheat, soy, and eggs [4].

Clinical symptoms of EoE vary depending on age. The most common symptoms in infants and toddlers are
Table 1. Clinical manifestations of EGIDs

\begin{tabular}{ll}
\hline EGID & Clinical symptoms \\
\hline EoE & $\begin{array}{l}\text { Vomiting, GERD-like symptoms, failure to thrive, } \\
\text { dysphagia, food impaction }\end{array}$ \\
EG & $\begin{array}{l}\text { Nausea, vomiting, retrosternal or epigastric pain, } \\
\text { dyspepsia, hematemesis/melaena (mucosal } \\
\text { involvement); outlet obstruction mimicking pyloric } \\
\text { stenosis (muscular involvement) }\end{array}$ \\
EGE & $\begin{array}{l}\text { Nausea, vomiting, abdominal pain, diarrhea, failure to } \\
\text { thrive/weight loss, protein loss or gastrointestinal } \\
\text { bleeding (mucosal involvement); obstructive symptoms, } \\
\text { intussusception, perforation (muscular involvement); } \\
\text { abdominal distention, ascites (serosal involvement) }\end{array}$ \\
EC & $\begin{array}{l}\text { Abdominal pain, tenesmus, diarrhea with mucus } \\
\text { and/or blood (mucosal involvement); volvulus, } \\
\text { intussusception, perforation (transmural involvement) }\end{array}$ \\
\hline &
\end{tabular}

feeding difficulties, in children vomiting and abdominal pain, while in adolescents, dysphagia and food impaction (Table 1).

Currently, there are no available specific biomarkers for the diagnosis of the disease; therefore, the diagnosis relies only on endoscopy and histology. The endoscopic findings in children with EoE vary from the presence of esophageal rings, furrows, and/or white exudates (Fig. 1a, b) to, less often, narrowing of the caliber of esophagus, although the presence of a normal esophagus does not exclude the diagnosis.

The main histological feature of EoE is dense but patchy eosinophilia of esophageal mucosa, usually associated with microabscesses, superficial layering, or extracellular eosinophil granules (Fig. 2a-c). The presence of at least 15 eosinophils per high-power field (eos/hpf), as peak value in at least one esophageal mucosal biopsy, is required for the histological definition of the disease (Table 2) [6]. The ESPGHAN recommends at least two to four esophageal biopsies to be taken from both the proximal and distal esophagus, regardless of the endoscopic appearance of the esophagus [7].

Esophageal eosinophilia is not an exclusive feature of EoE and, therefore, differential diagnosis should include other diseases that are associated with esophageal eosinophilia (Table 3).

The treatment of EoE has the following goals: (i) to achieve clinical and histological remission of the disease, (ii) to maintain remission, and (iii) to avoid iatrogenic damage. The efficacy of the treatment relies on demon- 
Table 2. Histological features of EGIDs [6, 20, 21, 26, 28, 29, 55, 56]

Eosinophilic ${ }^{1}$ infiltration of the lamina propria and/or submucosa, muscularis propria, or serosa

- As eosinophilic inflammation is patchy, peak eosinophilic counts for the diagnosis of EGIDs are necessary

The following numbers of eos/hpf have been proposed for the histological diagnosis of EGIDs, but for EGIDs beyond the esophagus, the numbers indicated below need to be confirmed by further studies in children:

EoE $\geq 15$ eos/hpf

$\mathrm{EG} \geq 30$ eos/hpf in $\geq 5 \mathrm{hpf}$

EGE $>50$ eos/hpf duodenum

EC $>50$ eos/hpf right colon; $>30$ eos/hpf left/transverse colon

- Other findings

Eosinophilic surface layering

Eosinophilic degranulation

Eosinophilic crypt abscesses

Basal zone hyperplasia

Dilated intercellular spaces

Lamina propria fibrosis

${ }^{1}$ The histological finding of increased numbers of eosinophils per high-power field (eos/hpf) in a biopsy specimen of the GI tract has no proven biological importance and cannot be used as the only tool for the differential diagnosis of EGIDs from other GI diseases

Table 3. Disorders associated with GI tract eosinophilia that should be included in the differential diagnosis with EGIDs

\begin{tabular}{ll}
\hline Esophagus & GERD \\
& Infections (herpes and candida) \\
& Esophageal achalasia \\
& Crohn's disease \\
& Connective tissue disorders \\
& Hypereosinophilic syndrome \\
& Drug sensitivity response \\
& Malignancy \\
& Celiac disease \\
& Helicobacter pylori infection \\
& Inflammatory bowel disease \\
& Connective tissue disorders \\
& Hypereosinophilic syndrome \\
Stomach & Infections (parasitic, amebic, fungal) \\
& Inflammatory bowel disease \\
& Connective tissue disorders \\
& Hypereosinophilic syndrome \\
& Vasculitis \\
& Malignancy \\
\hline
\end{tabular}

stration of histological and not only of clinical remission. The treatment strategies include elimination diet and/or drugs (proton pump inhibitors [PPIs] or steroids).

Three elimination diets have been used for treating EoE: (1) exclusive enteral nutrition with an amino acidbased formula (AAF); (2) empiric elimination diet; (3) targeted elimination diet. Exclusive enteral nutrition with an AAF consists of complete removal of food allergens from the diet substituted by a hypoallergenic formula based on amino acids and is reported to induce remission in up to $90 \%$ of adults and children with EoE [8]. Due to the high cost and the big number of endoscopies needed during food re-introduction, however, AAF is reserved for young infants with multiple food allergies, particularly in patients who do not respond or do not wish to follow a strict diet with multiple food elimination [7]. The targeted elimination diet is based on removal of foods detected with specific allergy testing and/or history of allergy and has limited place in the treatment of EoE, inducing remission in $45.5 \%$ of patients, because allergy testing cannot demonstrate the causative foods of the disease but only food sensitization [8]. The empiric elimination diet is based on removing the food allergens that have shown to strongly correlate with EoE from the diet. The six-food elimination diet (eliminating dairy products, soy, eggs, wheat, peanuts, fish, and shellfish) is the most studied empiric elimination diet. It has been reported to be highly effective in treating EoE, achieving histological remission in 75\% of patients with EoE [8]. Recently, the four-food elimination diet, avoiding cow's milk, wheat, eggs, and legumes, was reported to achieve histological remission in 71\% of children with EoE [9], while an even more recent study showed that a step-up diet strategy in treating childhood EoE is feasible: the two-food elimination diet restricting animal milk and gluten-containing cereals achieved remission in $40 \%$ of the patients with EoE, while the four-food elimination diet achieved remission in $52 \%$ and the six-food elimination diet in $65 \%$. Amongst responders to a two-food elimination diet, the most common food triggers were animal milk and gluten-containing cereals (15\%), while animal milk alone or gluten-containing cereals alone were reported in 60 and $25 \%$ of patients, respectively [10].

Based on the above findings, many clinicians prefer the step-up approach, eliminating, initially, two foods (dairy products and eggs or gluten-containing cereals), upgrading to four- and to six-food elimination in nonresponders, reserving the exclusive enteral nutrition with AAF for highly selected children. The above step-up di- 
Fig. 1. a, b Eosinophilic esophagitis (endoscopy). a Trachealization of the esophagus. b White exudates.
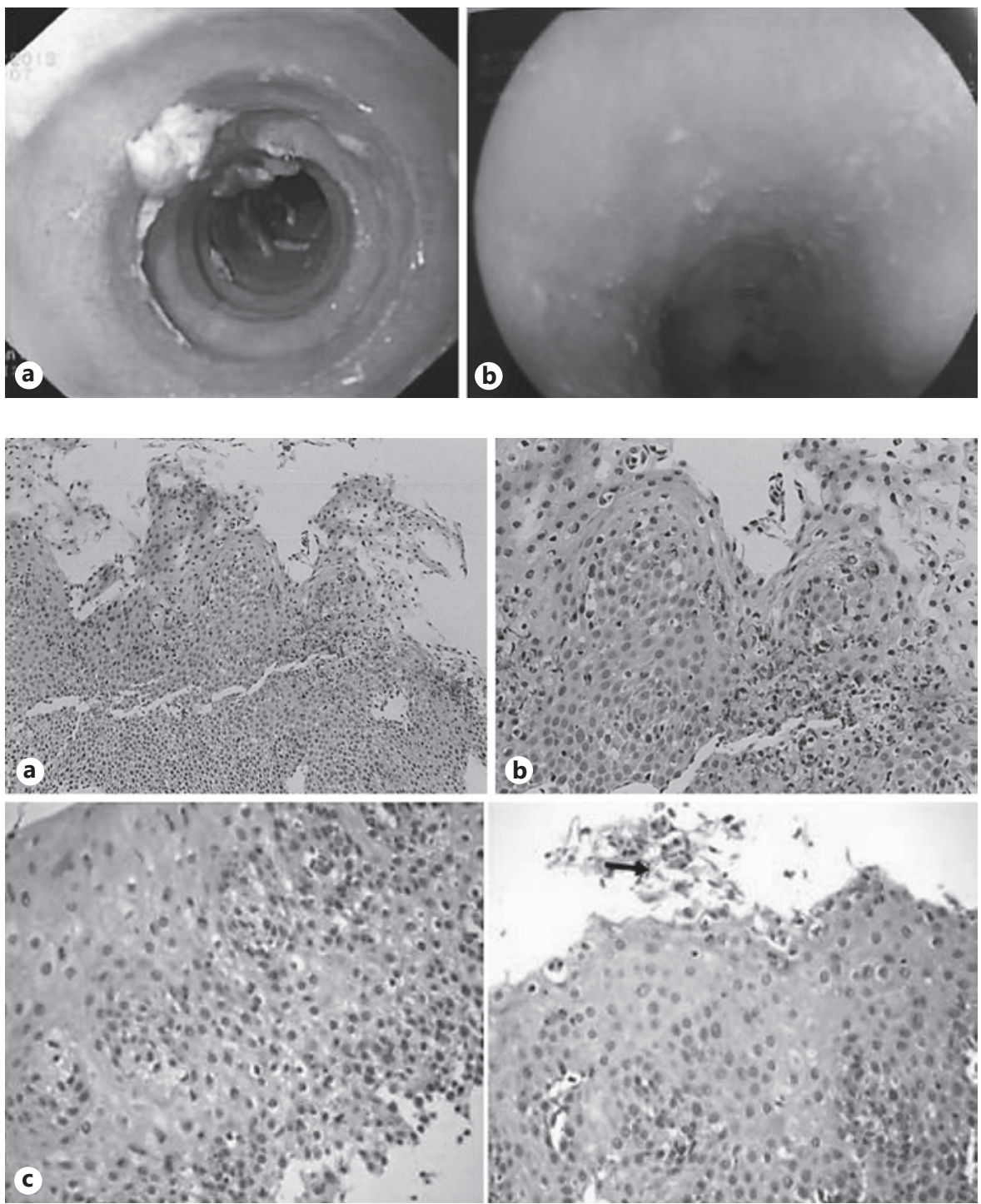

Fig. 2. a-c Eosinophilic esophagitis (histology). a Abscesses of eosinophils in the upper layers of the squamous epithelium. Mild /moderate hyperplasia of the basal layer. b Abscesses of eosinophils in the upper layers of the squamous epithelium. Severe degranulation of eosinophils. Moderate spongiosis. c Abscesses of eosinophils in the upper layers of the squamous epithelium (arrow). children with EoE. PPIs have acid-suppressive but also anti-inflammatory effects and therefore may induce remission in $45 \%$ of patients with EoE [11], probably with a milder form of the disease. The recommended dose of PPIs is $1 \mathrm{mg} / \mathrm{kg}$ per dose, twice daily with the maximum dose reaching the adult dose of $20-40 \mathrm{mg}$ once or twice daily depending on the patient and PPI. Oral systemic steroids are highly effective in inducing clinical and histological remission in children with EoE as early as at 2-4 weeks. Considering, however, the risks associated with the chronic use of oral systemic steroids, their use is considered only when immediate relief of the patient's symptoms such as severe dysphagia, weight loss, or esophageal stenosis, is needed. The effective dose for eliminating 
Fig. 3. Eosinophilic gastritis (histology). Aggregates of eosinophils near the muscularis mucosa (small arrow). Eosinophilic infiltration of pyloric glands (long arrow). Fig. 4. Eosinophilic gastritis (endoscopy). Gastric antral erosions and ulcers in a child with eosinophilic gastroenteritis presenting with epigastric pain and iron deficiency anemia.
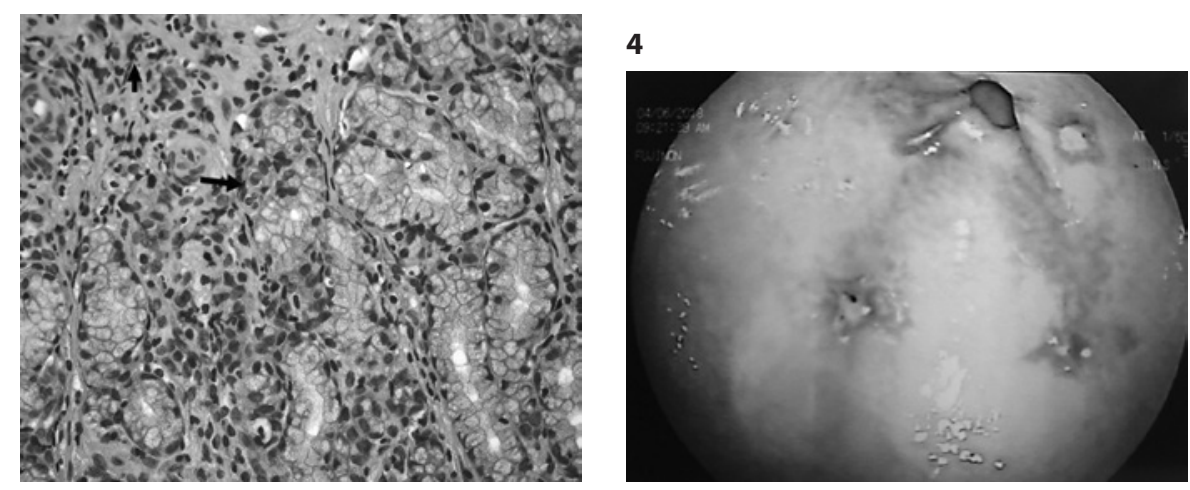

clinical symptoms and histological abnormalities is $1-2$ $\mathrm{mg} / \mathrm{kg} /$ day of prednisone with the maximum dose reaching 40-60 mg.

Topical steroids (swallowed fluticasone propionate and oral viscous budesonide) were assessed in patients with EoE and proved efficacy in achieving histological remission in patients with EoE as well as in maintaining remission. Fluticasone propionate is sprayed into the mouth with the lips sealed around the device and the patient is advised not to drink or eat for the next $30 \mathrm{~min}$. This drug was reported to induce remission in 50\% [12] to $91 \%$ of the patients [13]. The suggested dosage ranges from 88 to $440 \mu \mathrm{g}$ twice to 4 times daily for children and from 440 to $880 \mu \mathrm{g}$ twice daily for adolescents/adults. Oral viscous budesonide is prepared by mixing a liquid solution of budesonide $1 \mathrm{mg} / 2 \mathrm{~mL}$ (the preparation used for inhalations) and $5 \mathrm{~g}$ of sucralose. The administration of this preparation achieves histological remission in $87 \%$ of children [14] and in $72 \%$ of adolescents and adults with EoE [15]. The recommended dosage of oral viscous budesonide is $1 \mathrm{mg}$ daily for children $<10$ years and $2 \mathrm{mg}$ daily for older children and adults [6]. Viscous topical slurry is more effective than nebulized steroid therapy for patients with EoE, as it provides increased concentration of the drug in the esophagus [16]. The main side effect of topical steroids is oral candidiasis, resolving following drug discontinuation. Drug titration should be initiated after confirming histological remission following symptom resolution with a repeat endoscopy at 4-12 weeks following drug introduction. Continuation of drug therapy is needed for several months to maintain remission, but the optimal regimen, dose, and duration still need to be determined.

Other drugs that have been tested for the treatment of EoE are sodium cromoglycate or montelukast, a leukotriene receptor antagonist, but they could not demonstrate any efficacy and thus are not recommended for treating
EoE. The same is true for immunomodulating drugs and biologics. The efficacy of monoclonal antibodies against IL-5 in children with EoE needs further evaluation, while anti-IgE monoclonal antibodies were effective in improving food tolerance but not in achieving histological remission [17]. Patients with severe dysphagia due to esophageal stenosis, who do not respond to medical treatment, have their symptoms relieved with endoscopic esophageal dilatation, which is performed in combination with medical treatment. Furthermore, some patients with EoE have seasonal exacerbations of the disease, caused by inhaled aeroallergens including pollens and moulds, and are often associated with food impaction [18]. In case of an established pattern of seasonal exacerbations in children with EoE, preventive measures using topical corticosteroids may be used.

In conclusion, EoE is a chronic, relapsing inflammatory disease of the esophagus which often requires prolonged therapy. Investigations and treatment need to be individualized. Further randomized studies to assess the efficacy of biological markers in disease diagnosis and prognosis, the most appropriate regimen, dosing, and duration of the maintenance therapy in different disease phenotypes, as well as the efficacy of novel agents for treating refractory disease, are urgently needed.

\section{Eosinophilic Gastritis}

EG is a clinical entity characterized by dense eosinophilic infiltration of the stomach wall. It is the second most common form of EGID after EoE, with a prevalence of about 6.3 patients per 100,000 individuals [19]. EG is found to be more prevalent among older age groups, with female predominance [19]. Furthermore, an association with documented allergic conditions was reported in $58.9 \%$ of children and in $33.6 \%$ of adults with EG [19]. 
Fig. 5. a, b Eosinophilic gastroenteritis (histology of duodenal biopsies). a Erosion and aggregates of eosinophils (long arrow). Eosinophilic infiltration of the Brunner glands (small arrow). b Aggregates of eosinophils at the deep part of the crypts with degranulation (long arrow). Eosinophilic infiltration of the crypts (small arrow).
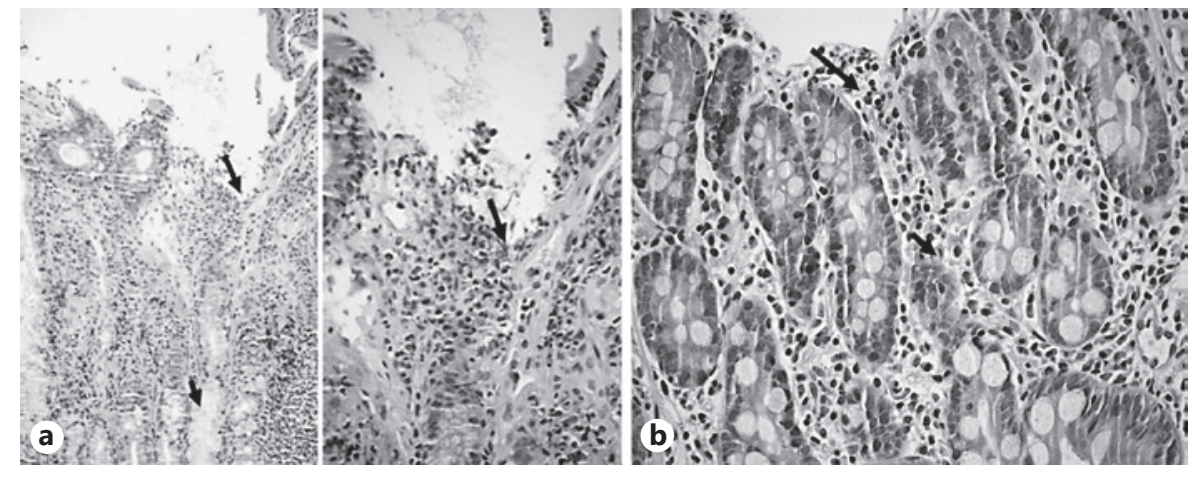

The histological criteria (Table 2) for the diagnosis of EG include the presence of dense eosinophilic inflammation of the gastric mucosa $[20,21]$, associated with eosinophil cryptitis, eosinophilic abscesses (Fig. 3), or the presence of eosinophils in the submucosa and muscularis mucosa. It has been proposed that $\geq 30 \mathrm{eos} / \mathrm{hpf}$ in $\geq 5 \mathrm{hpf}$ are needed for the histological diagnosis of EG [20,21], but this has to be confirmed by further studies in children. Furthermore, apart from the eosinophilic activation, mast cells and FOXP3-positive lymphocytes have also been reported to be activated in EG [22].

The clinical manifestations depend on the gastric layer involved in the inflammatory process. Of these, the mucosal involvement is the most common, presenting clinically with epigastric pain, nausea, vomiting, and early satiety, while the laboratory findings include peripheral eosinophilia, hypoalbuminemia, and iron deficiency anemia [23]. Furthermore, case reports in infants have attributed pyloric stenosis to EG [24] and the same was true for isolated giant ulcers resistant to PPIs, mainly in adolescent girls [25].

Endoscopy reveals nodules in the gastric mucosa, erythema and ulceration or erosions (Fig. 4), even though in some cases, the mucosa may appear completely normal [23].

Corticosteroids in their oral or topical form seem to be an effective therapy of EG. Case reports have suggested the off-label use of budesonide in the form of capsules dissolved in water, to target the upper GI tract [26], while others indicated that fluticasone led to a resolution of eosinophilic gastric inflammation when used initially to treat EoE [27].

In some children with EG, food antigen restriction may resolve both the symptoms and the histology abnormalities. Ko et al. [23] reported, in a retrospective study, that $82 \%$ of the patients with EG achieved clinical and $78 \%$ histological remission following elimination diet (elemental diet, elimination diet excluding milk, egg, wheat,

Eosinophilic Gastrointestinal Diseases in Childhood soy, peanut/tree nuts, fish/shellfish, and red meat from the diet and also empiric avoidance of 1-3 foods). However, due to the fact that only up to 5 children per each dietary treatment group underwent a histological evaluation, no strict conclusions could be drawn from the above study [23]. An interesting finding in that study, however, was that although $86 \%$ of patients were found to be sensitized to several foods using skin prick tests or serum analyses, no correlation was found between response to dietary therapy and food sensitization [23].

In conclusion, EG is a chronic, relapsing disease, although its long-term outcome and clinical consequences are poorly defined. Elimination diet and/or steroids are considered as the first-line therapy for induction of remission. More studies are needed to assess the efficacy of different therapeutic approaches for maintenance.

\section{Eosinophilic Gastroenteritis}

EGE is a rare inflammatory disorder, characterized by eosinophilic infiltration of the stomach and small intestine (Fig. 5a, b), and in some cases, the esophagus and colon without any other known causes of GI eosinophilic inflammation [1].

The diagnosis of EGE is based, histologically, on the presence of a dense eosinophilic inflammation of the intestine often associated with eosinophilic degranulation $[21,28,29]$. It has been proposed that $>50$ eos/hpf are needed for the histological diagnosis of EGE (Table 2), but this number has to be confirmed by further studies in children. The prevalence of EGE is approximately 5.1 per 100,000 individuals [30]. EGE is more common among children of age $<5$ years [19]. It presents clinically with abdominal pain, abdominal distention, nausea, vomiting, diarrhea, weight loss, and sometimes with serious complications such as intestinal obstruction and perforation $[31,32]$. 


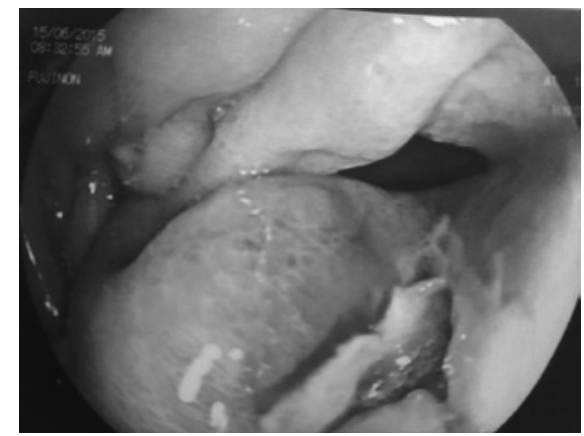

Fig. 6. Eosinophilic gastroenteritis (endoscopy). Duodenal giant ulcer in a child with eosinophilic gastroenteritis presenting with hematemesis.

Fifty-two percent of children and $41.8 \%$ of adults with EGE have a history of an allergic disease [19]. The clinical features of EGE vary according to the location, extent, and layer(s) involved in the inflammatory process $[33,34]$. Mucosal disease may present with abdominal pain, nausea, vomiting, early satiety, and diarrhea, often associated with failure to thrive or weight loss. Patients with diffuse small intestine disease may develop malabsorption, anemia, and hypoalbuminemia [34]. In case of involvement of the muscular layer, eosinophilic inflammation may cause wall thickening, impaired motility, and intestinal obstruction presenting clinically with nausea, vomiting, and abdominal distention that may lead to perforation [35]. Subserosal disease is presenting with ascites [35].

As for the other EGIDs, except for EoE, there are no consensus diagnostic criteria for EGE. The diagnosis is based on the presence of GI symptoms (Table 1), eosinophilic infiltration of the GI tract (Table 2), or eosinophilic ascites on condition of the exclusion of other causes of intestinal eosinophilia (Table 3).

Laboratory findings include peripheral blood eosinophilia in $20-80 \%$ of patients [35] with an average absolute eosinophil count of 1,000 cells [36]. Mucosal and subserosal EGE are characterized by higher eosinophil counts compared to muscular EGE with an average absolute eosinophil count of 2,000 and 8,000 cells/ $\mu \mathrm{L}$, respectively. Serum albumin may be low due to protein-losing enteropathy and iron deficiency anemia may occur due to impaired iron absorption and occult GI bleeding, especially in the mucosal subtype of the disease [34]. Imaging studies in patients with the muscular subtype of the disease may reveal irregular narrowing of the gut lumen. In patients with serosal disease and ascites, ascetic fluid analysis shows a marked elevation of the eosinophil counts [34].
Endoscopic findings of EGE appear to be nonspecific and range from nodular or polypoid appearance of the mucosa to erythema, friability, and occasional ulcers (Fig. 6) or erosions [37].

Biopsies should be taken from both normal and abnormal appearing mucosa since even a normal appearing mucosa can be infiltrated by eosinophils [38]. At least 4-5 biopsies should be taken from the stomach and the small intestine, including from the macroscopically normal mucosa. Patients with muscular or subserosal type of EGE can have normal mucosal biopsies. In that case, laparoscopic full-thickness biopsy should be performed to confirm the diagnosis. Laparoscopic full-thickness biopsy should also be performed in patients with intestinal wall thickening and/or obstruction to exclude a possible underlying malignancy. The differential diagnosis of EGE includes several diseases associated with eosinophilic inflammation of the GI tract (Table 3).

Due to the rarity of EGE, there are only limited data concerning the best treatment option based on the severity of symptoms. Reed et al. [39] assessed the efficacy of various treatment modalities including dietary therapy, corticosteroids, mast cell inhibitors, $\mathrm{H} 2$ antagonists, and leukotriene receptor antagonists in 44 patients with EGE, including children, for an average of 26.2 months with $76 \%$ of patients needing more than one treatment option. When all treatment modalities were included, $60 \%$ of patients achieved clinical remission, while $51 \%$ achieved histological remission [39].

In symptomatic patients and in those with symptoms of malabsorption, an initial therapeutic approach could involve elimination diet [40, 41]. Gonsalves et al. [42], in a study involving adults with EGE, found a significant reduction in symptoms, complete histological remission, improvement in endoscopic findings, and normalization of peripheral eosinophilia after the six-food elimination diet or AAF within a period of 6 weeks. Katz et al. [43] reported that infants below 1 year of age, but not older children, responded well to cow's milk protein elimination diet.

Whenever dietary therapy is used, it should be supervised by an expert to assure patients' compliance and avoid nutritional compromise. Food reintroduction starts slowly, from least to most allergenic foods, following demonstration of histological remission of the disease.

Evidence to support the use of glucocorticoids in EGE is based on case series $[37,44]$. Glucocorticoids are known to decrease the chemotaxis of inflammatory cells including eosinophils, decrease the release of eotaxins and other inflammatory mediators, and reduce permeability. The 
use of steroids is usually associated with a rapid improvement (within 2 weeks), regardless of the depth of inflammation through the bowel wall [44] and, therefore, a rapid tapering over the following 2-4 weeks is suggested. Fibrosis is much less common in EGE compared to EoE; therefore, oral steroids can be used at a minimum effective dose, e.g., $0.5-1 \mathrm{mg} / \mathrm{kg}$ with a maximum dose of 40 $\mathrm{mg}$, while the whole duration of the treatment with oral steroids should not exceed 6 weeks. It should be noted, however, that discontinuation of steroids is often associated with disease relapse [45].

Furthermore, the use of oral budesonide suspension or the ileal releasing budesonide enteric capsules [46] crashed
In the absence of reliable biological markers, the diagnosis of EGIDs is based on the histological findings in biopsy specimens taken from the GI tract of symptomatic children, a quite challenging issue given the absence of strict histological criteria for EGID (apart from EoE) diagnosis
EGE had long-lasting remission. The most common complication of the disease is GI obstruction, while fatal outcomes are fortunately rare.

To conclude, EGE is a rare inflammatory GI disease. Clinical symptoms depend on the segment of the GI tract involved as well as the extent and depth of eosinophilic infiltration through the bowel wall. The diagnosis is based on the finding of dense eosinophilic infiltration of the stomach and/or small intestine in the clinical context. First-line treatment for induction of remission includes elimination diet and/or steroids, while maintenance therapy is tailored to the individual. Long-term follow-up studies to determine the longand dissolved in water or juice in order to reach upper GI segments has been beneficial in some patients with EGE involving the antrum and the small bowel [26], while the standard preparations are useful in case of ileum involvement. Other drugs have also been evaluated for the treatment of EGE. Sodium cromoglycate that inhibits the release of mast cell mediators and the antigen absorption in the gut or montelukast, that is a leukotriene antagonist, do not manage to induce clinical or histological remission when used as sole therapy [39]. Ketotifen (an H1-antihistamine and mast cell stabilizer), in small series of patients, improved clinical symptoms and tissue eosinophilia [47]. Humanized anti-IL-5 antibody was reported in a pilot study, to reduce peripheral and tissue eosinophilia but failed to improve symptoms, while it was associated with histological relapse after drug discontinuation [48]. Finally, thiopurines were reported to be effective in refractory cases [49], while omalizumab, an anti-IgE monoclonal antibody, was associated with improvement in symptoms but not of histological abnormalities [50]. Surgical treatment should be avoided and limited only to cases of persisted pyloric or small bowel obstruction despite treatment.

The natural history of EGE is poorly defined. Pineton de Chambrun et al. [51] reported in 43 patients with EGIDs that 18 (42\%) had no relapses after initial diagnosis, 17 (37\%) had multiple relapses, while 9 (21\%) developed chronic disease. In patients with recurring disease, the intervals of remission between relapses ranged from months to years. Similarly, Reed et al. [39] claimed that only one-third of the pediatric and adult patients with term outcomes of different disease phenotypes, are urgently needed.

\section{Eosinophilic Colitis}

EC is a rare inflammatory GI disorder characterized clinically by symptoms related to colonic dysfunction and colon biopsies histology indicating excessive accumulation of eosinophils [52]. The prevalence of EC is estimated to be about 2.1 per 100,000 individuals [30], with no prominent differences related to age or gender [19, 30], although some authors claimed a predominance of males [53]. Associated atopy has been reported in $52.0 \%$ of pediatric patients and in $35.9 \%$ of adults with EC [19].

Common histological abnormalities found in colonic biopsies from patients with EC include increased eosinophilic density (Fig. 7), eosinophilic cryptitis or crypt abscesses, crypts architecture impairment, increased intraepithelial eosinophils, and/or the presence of eosinophils in the muscularis mucosa and submucosa [54].The histological criteria for diagnosing EC in adults include the presence of at least $100 \mathrm{eos} / \mathrm{hpf}$ in the cecum and ascending colon, 84 eos/hpf in the transverse and descending colon, and 64 eos/hpf in the rectosigmoid area [21]. In children, there is no consensus on the cutoff number of eosinophils for EC diagnosis, although numbers above 50 eos/hpf [55] depending on the site of the colon, in the clinical context, may aid the EC diagnosis, but this needs to be confirmed by further studies in children. 


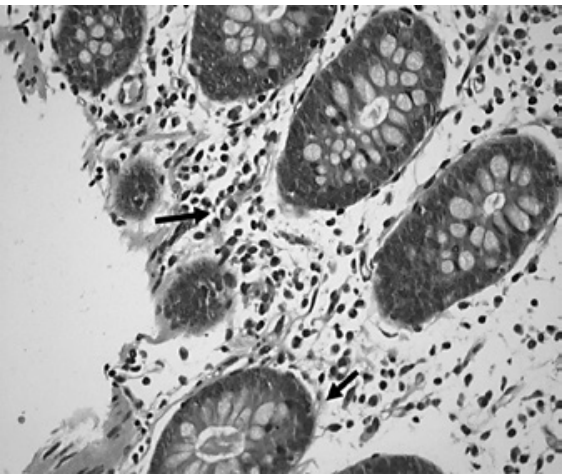

Fig. 7. Eosinophilic colitis (histology). Aggregates of eosinophils at the deep part of the colonic crypts with degranulation (long arrow). Eosinophilic infiltration of colonic crypts (small arrow).

The most common symptoms of EC (Table 1) are abdominal pain and diarrhea, while volvulus or intussusception have also been reported [56]. Endoscopic findings include mucosal granularity, erythema, the presence of ulcers or erosions, and/or white exudates.

EC is thought to be an enigmatic disease since several studies have documented the presence of eosinophilic infiltration in colonic biopsies of patients with allergy or inflammatory bowel disease [57]. The presence of inflammatory cell populations in combination with excessive eosinophilic infiltration may raise the suspicion of inflammatory bowel disease, while the presence of deposits of both IgE and tryptase in perineural areas may raise the suspicion of EC [58]. Furthermore, colonic tissue eosinophilia can also be found in patients with other disorders that need to be considered in the differential diagnosis (Table 3).

The most effective treatment of EC are corticosteroids. It should be noted, however, that a subgroup of patients, mainly infants or young children, may also benefit from the elimination diet [59], while the use of anti-TNF biological drugs such as infliximab or adalimumab may achieve long-lasting remission in refractory disease [60].

\section{Conclusions}

EGIDs are emerging chronic, inflammatory disorders in childhood with largely unknown long-term consequences. Their clinical presentation depends on the GI layer involved, the extend of the inflammation in the GI tract, and the depth of the eosinophilic inflammation through the bowel wall. In the absence of reliable biological markers, the diagnosis of EGIDs is based on the histological findings in biopsy specimens taken from the GI tract of symptomatic children, a quite challenging issue given the absence of strict histological criteria for EGID (apart from EoE) diagnosis. The exclusion of secondary causes of GI inflammation is critical. Therapeutic approaches are based mainly on case reports and small series of patients. First-line therapy includes elimination diets and/or drugs that are chosen depending on the involved GI segment. International, consensus recommendations on EGID diagnosis are urgently needed, in order to facilitate high-quality randomized controlled trials to assess the efficacy of various treatment approaches for achieving and maintaining remission while ensuring normal growth and quality of life.

\section{Acknowledgements}

The authors thank Dr. Kalliopi Stefanaki, the Chief of the Pathology Department of the Children's Hospital "Agia Sofia," for providing pictures of the histology slides from the GI biopsies of our patients with EGID for the needs of this review.

\section{Disclosure Statement}

The writing of this article was supported by Nestlé Nutrition Institute. A. Papadopoulou has received honorarium for the writing of this article, research grants from Biogaia and Abbvie, speaker's honorariums from Abbvie, Nestlé, Nutricia, Friesland and Vian, and has served as member of advisory board for Adare Pharmaceuticals. E. Koutri declares no other conflicts of interest.

\section{References}

1 Furuta GT, Forbes D, Boey C, Dupont C, Putnam P, Roy S, et al.; Eosinophilic Gastrointestinal Diseases Working Group. Eosinophilic gastrointestinal diseases (EGIDs). J Pediatr Gastroenterol Nutr. 2008 Aug;47(2):234-8.

2 Liacouras CA, Furuta GT, Hirano I, Atkins D, Attwood SE, Bonis PA, et al. Eosinophilic esophagitis: updated consensus recommendations for children and adults. J Allergy Clin Immunol. 2011;128:3-20.
3 Arias Á, Pérez-Martínez I, Tenías JM, Lucendo AJ. Systematic review with meta-analysis: the incidence and prevalence of eosinophilic oesophagitis in children and adults in population-based studies. Aliment Pharmacol Ther. 2016 Jan;43(1):3-15.
4 Spergel JM, Brown-Whitehorn TF, Cianferoni A, Shuker M, Wang ML, Verma R, et al. Identification of causative foods in children with eosinophilic esophagitis treated with an elimination diet. J Allergy Clin Immunol. 2012 Aug;130(2):461-7.e5.

5 Mishra A, Hogan SP, Brandt EB, Rothenberg ME. An etiological role for aeroallergens and eosinophils in experimental esophagitis. J Clin Invest. 2001 Jan;107(1):83-90. 
6 Furuta GT, Liacouras CA, Collins MH, Gupta SK, Justinich C, Putnam PE, et al.; First International Gastrointestinal Eosinophil Research Symposium (FIGERS) Subcommittees. Eosinophilic esophagitis in children and adults: a systematic review and consensus recommendations for diagnosis and treatment. Gastroenterology. 2007 Oct;133(4):1342-63.

7 Papadopoulou A, Koletzko S, Heuschkel R, Dias JA, Allen KJ, Murch SH, et al.; ESPGHAN Eosinophilic Esophagitis Working Group and the Gastroenterology Committee. Management guidelines of eosinophilic esophagitis in childhood. J Pediatr Gastroenterol Nutr. 2014 Jan;58(1):107-18.

8 Arias A, González-Cervera J, Tenias JM, Lucendo AJ. Efficacy of dietary interventions for inducing histologic remission in patients with eosinophilic esophagitis: a systematic review and meta-analysis. Gastroenterology. 2014 Jun;146(7):1639-48.

9 Kagalwalla AF, Wechsler JB, Amsden K, Schwartz S, Makhija M, Olive A, et al. Efficacy of a 4-Food Elimination Diet for Children With Eosinophilic Esophagitis. Clin Gastroenterol Hepatol. 2017 Nov; 15(11):16981707.e7.

10 Molina-Infante J, Arias Á, Alcedo J, GarciaRomero R, Casabona-Frances S, Prieto-Garcia A, et al. Step-up empiric elimination diet for pediatric and adult eosinophilic esophagitis: The 2-4-6 study. J Allergy Clin Immunol. 2018 Apr;141(4):1365-72.

11 Lucendo AJ, Arias Á, Molina-Infante J. Efficacy of Proton Pump Inhibitor Drugs for Inducing Clinical and Histologic Remission in Patients With Symptomatic Esophageal Eosinophilia: A Systematic Review and MetaAnalysis. Clin Gastroenterol Hepatol. 2016 Jan;14(1):13-22.e1.

12 Konikoff MR, Noel RJ, Blanchard C, Kirby C, Jameson SC, Buckmeier BK, et al. A randomized, double-blind, placebo-controlled trial of fluticasone propionate for pediatric eosinophilic esophagitis. Gastroenterology. 2006 Nov;131(5):1381-91.

13 Helou EF, Simonson J, Arora AS. 3-yr-followup of topical corticosteroid treatment for eosinophilic esophagitis in adults. Am J Gastroenterol. 2008 Sep;103(9):2194-9.

14 Dohil R, Newbury R, Fox L, Bastian J, Aceves $S$. Oral viscous budesonide is effective in children with eosinophilic esophagitis in a randomized, placebo-controlled trial. Gastroenterology. 2010 Aug;139(2):418-29.

15 Straumann A, Conus S, Degen L, Felder S, Kummer M, Engel H, et al. Budesonide is effective in adolescent and adult patients with active eosinophilic esophagitis. Gastroenterology. 2010 Nov;139(5):1526-37.

16 Dellon ES, Sheikh A, Speck O, Woodward K, Whitlow AB, Hores JM, et al. Viscous topical is more effective than nebulized steroid therapy for patients with eosinophilic esophagitis. Gastroenterology. 2012 Aug;143(2):321-4.e1.
17 Rocha R, Vitor AB, Trindade E, Lima R, Tavares $\mathrm{M}$, Lopes $\mathrm{J}$, et al. Omalizumab in the treatment of eosinophilic esophagitis and food allergy. Eur J Pediatr. 2011 Nov;170(11): 1471-4.

18 Moawad FJ, Veerappan GR, Lake JM, Maydonovitch CL, Haymore BR, Kosisky SE, et al. Correlation between eosinophilic oesophagitis and aeroallergens. Aliment Pharmacol Ther. 2010 Feb;31(4):509-15.

19 Jensen ET, Martin CF, Kappelman MD, Dellon ES. Prevalence of Eosinophilic Gastritis, Gastroenteritis, and Colitis: Estimates From a National Administrative Database. J Pediatr Gastroenterol Nutr. 2016 Jan;62(1):36-42.

20 Lwin T, Melton SD, Genta RM. Eosinophilic gastritis: histopathological characterization and quantification of the normal gastric eosinophil content. Mod Pathol. 2011 Apr; 24(4):556-63

21 Collins MH, Capocelli K, Yang GY. Eosinophilic gastrointestinal disorders pathology. Front Med (Lausanne). 2018 Jan;4:261.

22 Caldwell JM, Collins MH, Stucke EM, Putnam PE, Franciosi JP, Kushner JP, et al. Histologic eosinophilic gastritis is a systemic disorder associated with blood and extragastric eosinophilia, TH2 immunity, and a unique gastric transcriptome. J Allergy Clin Immunol. 2014 Nov; 134(5):1114-24.

23 Ko HM, Morotti RA, Yershov O, Chehade M. Eosinophilic gastritis in children: clinicopathological correlation, disease course, and response to therapy. Am J Gastroenterol. 2014 Aug;109(8):1277-85.

24 Snyder JD, Rosenblum N, Wershil B, Goldman H, Winter HS. Pyloric stenosis and eosinophilic gastroenteritis in infants. J Pediatr Gastroenterol Nutr. 1987 Jul-Aug;6(4):543-7.

25 Kristopaitis T, Neghme C, Yong SL, Chejfec G, Aranha G, Keshavarzian A. Giant antral ulcer: a rare presentation of eosinophilic gastroenteritis-case report and review of the literature. Am J Gastroenterol. 1997 Jul;92(7): 1205-8.

26 Prussin C. Eosinophilic gastroenteritis and related eosinophilic disorders. Gastroenterol Clin North Am. 2014 Jun;43(2):317-27.

27 Ammoury RF, Rosenman MB, Roettcher D, Gupta SK. Incidental gastric eosinophils in patients with eosinophilic esophagitis: do they matter? J Pediatr Gastroenterol Nutr. 2010 Dec;51(6):723-6.

28 Lucendo AJ, Arias A. Eosinophilic gastroenteritis: an update. Expert Rev Gastroenterol Hepatol. 2012 Sep;6(5):591-601.

29 Ingle SB, Hinge Ingle CR. Eosinophilic gastroenteritis: an unusual type of gastroenteritis. World J Gastroenterol. 2013 Aug;19(31): 5061-6.

30 Mansoor E, Saleh MA, Cooper GS. Prevalence of Eosinophilic Gastroenteritis and Colitis in a Population-Based Study, From 2012 to 2017. Clin Gastroenterol Hepatol. 2017 Nov; 15(11):1733-41.
31 Shweiki E, West JC, Klena JW, Kelley SE, Colley AT, Bross RJ, et al. Eosinophilic gastroenteritis presenting as an obstructing cecal mass-a case report and review of the literature. Am J Gastroenterol. 1999 Dec;94(12): 3644-5.

32 Tran D, Salloum L, Tshibaka C, Moser R. Eosinophilic gastroenteritis mimicking acute appendicitis. Am Surg. 2000 Oct;66(10): 990-2.

33 Rothenberg ME. Eosinophilic gastrointestinal disorders (EGID). J Allergy Clin Immunol. 2004 Jan;113(1):11-28.

34 Klein NC, Hargrove RL, Sleisenger MH, Jeffries GH. Eosinophilic gastroenteritis. Medicine (Baltimore). 1970 Jul;49(4):299-319.

35 Talley NJ, Shorter RG, Phillips SF, Zinsmeister AR. Eosinophilic gastroenteritis: a clinicopathological study of patients with disease of the mucosa, muscle layer, and subserosal tissues. Gut. 1990 Jan;31(1):54-8.

36 Chang JY, Choung RS, Lee RM, Locke GR 3rd, Schleck CD, Zinsmeister AR, et al. A shift in the clinical spectrum of eosinophilic gastroenteritis toward the mucosal disease type. Clin Gastroenterol Hepatol. 2010 Aug;8(8): 669-75.

37 Chen MJ, Chu CH, Lin SC, Shih SC, Wang TE. Eosinophilic gastroenteritis: clinical experience with 15 patients. World J Gastroenterol. 2003 Dec;9(12):2813-6.

38 Lee M, Hodges WG, Huggins TL, Lee EL. Eosinophilic gastroenteritis. South Med J. 1996 Feb;89(2):189-94.

39 Reed C, Woosley JT, Dellon ES. Clinical characteristics, treatment outcomes, and resource utilization in children and adults with eosinophilic gastroenteritis. Dig Liver Dis. 2015 Mar;47(3):197-201.

40 Justinich C, Katz A, Gurbindo C, Lepage G, Chad Z, Bouthillier L, et al. Elemental diet improves steroid-dependent eosinophilic gastroenteritis and reverses growth failure. J Pediatr Gastroenterol Nutr. 1996 Jul;23(1): $81-5$.

41 Chehade M, Magid MS, Mofidi S, NowakWegrzyn A, Sampson HA, Sicherer SH. Allergic eosinophilic gastroenteritis with protein-losing enteropathy: intestinal pathology, clinical course, and long-term follow-up. J Pediatr Gastroenterol Nutr. 2006 May;42(5): 516-21.

42 Gonsalves N, Doerfler B, Yang G, Hirano I. S1861 A Prospective Clinical Trial of Six Food Elimination Diet or Elemental Diet in the Treatment of Adults with Eosinophilic Gastroenteritis. Gastroenterology. 2009; 136(5):A-280.

43 Katz AJ, Twarog FJ, Zeiger RS, Falchuk ZM. Milk-sensitive and eosinophilic gastroenteropathy: similar clinical features with contrasting mechanisms and clinical course. J Allergy Clin Immunol. 1984 Jul;74(1):72-8 571

44 Lee CM, Changchien CS, Chen PC, Lin DY, Sheen IS, Wang CS, et al. Eosinophilic gastroenteritis: 10 years experience. Am J Gastroenterol. 1993 Jan;88(1):70-4. 
45 Choi JS, Choi SJ, Lee KJ, Kim A, Yoo JK, Yang $\mathrm{HR}$, et al. Clinical Manifestations and Treatment Outcomes of Eosinophilic Gastroenteritis in Children. Pediatr Gastroenterol Hepatol Nutr. 2015 Dec;18(4):253-60.

46 Tan AC, Kruimel JW, Naber TH. Eosinophilic gastroenteritis treated with non-entericcoated budesonide tablets. Eur J Gastroenterol Hepatol. 2001 Apr;13(4):425-7.

47 Melamed I, Feanny SJ, Sherman PM, Roifman CM. Benefit of ketotifen in patients with eosinophilic gastroenteritis. Am J Med. 1991 Mar;90(3):310-4.

48 Prussin C, James S, Huber M, Klion A, Metcalfe D. Pilot study of anti-IL-5 in eosinophilic gastroenteritis. J Allergy Clin Immunol. 2003;111(2):S275

49 Redondo-Cerezo E, Cabello MJ, González Y, Gómez M, García-Montero M, de Teresa J. Eosinophilic gastroenteritis: our recent experience: one-year experience of atypical onset of an uncommon disease. Scand J Gastroenterol. 2001 Dec;36(12):1358-60.
50 Foroughi S, Foster B, Kim N, Bernardino LB, Scott LM, Hamilton RG, et al. Anti-IgE treatment of eosinophil-associated gastrointestinal disorders. J Allergy Clin Immunol. 2007 Sep;120(3):594-601.

51 Pineton de Chambrun G, Gonzalez F, Canva JY, Gonzalez S, Houssin L, Desreumaux P, et al. Natural history of eosinophilic gastroenteritis. Clin Gastroenterol Hepatol. 2011 Nov; 9(11):950-956.e1.

52 Turner KO, Sinkre RA, Neumann WL, Genta RM. Primary Colonic Eosinophilia and Eosinophilic Colitis in Adults. Am J Surg Pathol. 2017 Feb;41(2):225-33.

53 Mark J, Fernando SD, Masterson JC, Pan Z, Capocelli KE, Furuta GT, et al. Clinical Implications of Pediatric Colonic Eosinophilia. Pediatr Gastroenterol Nutr. 2018 May;66(5): $760-6$.

54 Gaertner WB, Macdonald JE, Kwaan MR, Shepela C, Madoff R, Jessurun J, et al. Eosinophilic colitis: university of Minnesota experience and literature review. Gastroenterol Res Pract. 2011;2011:857508.

55 DeBrosse CW, Case JW, Putnam PE, Collins $\mathrm{MH}$, Rothenberg ME. Quantity and distribution of eosinophils in the gastrointestinal tract of children. Pediatr Dev Pathol. 2006 MayJun;9(3):210-8.
56 Okpara N, Aswad B, Baffy G. Eosinophilic colitis. World J Gastroenterol. 2009 Jun; 15(24):2975-9.

57 Morgenstern S, Brook E, Rinawi F, Shamir R, Assa A. Tissue and peripheral eosinophilia as predictors for disease outcome in children with ulcerative colitis. Dig Liver Dis. 2017 Feb;49(2):170-4.

58 Torrente F, Barabino A, Bellini T, Murch SH. Intraepithelial lymphocyte eotaxin-2 expression and perineural mast cell degranulation differentiate allergic/eosinophilic colitis from classic IBD. J Pediatr Gastroenterol Nutr. 2014 Sep;59(3):300-7.

59 Yang M, Geng L, Chen P, Wang F, Xu Z, Liang $\mathrm{C}$, et al. Effectiveness of dietary allergen exclusion therapy on eosinophilic colitis in Chinese infants and young children $\leq 3$ years of age. Nutrients. 2015 Mar;7(3):1817-27.

60 Turner D, Wolters VM, Russell RK, Shakhnovich V, Muise AM, Ledder O, et al. AntiTNF, infliximab, and adalimumab can be effective in eosinophilic bowel disease. J Pediatr Gastroenterol Nutr. 2013 May;56(5):492-7. 